\title{
Acción in vitro de diez plantas medicinales sobre diez cepas diferentes de Streptococcus pneumoniae
} In vitro activity of ten medicinal plants on ten Streptococcus pneumoniae strains

\author{
Guevara Duncan JM¹, Fuertes Ruitón $C^{2}$, Valencia Bazalar E ${ }^{3}$, Guevara Granados JMM ${ }^{1,3}$, \\ Zerpa Larrauri $\mathrm{R}^{1,4}$, Béjar Castillo V ${ }^{1}$, Patiño Gabriel L ${ }^{4}$, Celis Salinas JC ${ }^{5}$ \\ 'Instituto de Medicina Tropical "Daniel A. Carrión”, Universidad Nacional Mayor de San Marcos, Lima, Perú. \\ ${ }^{2}$ Instituto de Ciencias Farmacéuticas y Recursos Naturales "Juan de Dios Guevara", Facultad de Farmacia y Bioquímica, UNMSM. \\ ${ }^{3}$ Hospital Nacional Daniel A. Carrión, Callao, Perú. \\ ${ }^{4}$ Instituto Nacional de Salud del Niño, Lima, Perú. \\ ${ }^{5}$ Hospital Nacional de lquitos, Perú.
}

\begin{abstract}
Resumen
Introducción: El portador sano es el principal diseminador de las infecciones neumocócicas por la nasofaringe. Una alternativa para combatirlo son las plantas medicinales. Objetivos: Determinar la efectividad de diez plantas medicinales frente a Streptococcus pneumoniae. Diseño: Estudio experimental in vitro. Material biológico: Plantas medicinales y cepas de Streptococcus pneumoniae. Intervenciones: Los extractos de 10 plantas medicinales fueron puestos en contacto in vitro con 10 cepas de Streptococcus pneumoniae. Principales medidas de resultados: Actividad de las plantas medicinales sobre las cepas de Streptococcus pneumoniae. Resultados: almendro y Bellaco caspi fueron las únicas plantas que dieron pequeño halo de inhibición con algunas cepas; con almendro se inhibió dos cepas y con Bellaco caspi, se inhibió siete cepas y tres resultaron resistentes. Estos resultados no tuvieron relación con el serotipo de neumococo ni con los antibióticos utilizados en los antibiogramas realizados previamente. Conclusiones: El Bellaco caspi podria ser una alternativa para atacar al neumococo en la nasofaringe. Pero, por haber presentado resistencia a tres cepas de Streptococcus pneumoniae, antes de usarlo sería conveniente desarrollar un antibiograma de los neumococos contra las plantas medicinales.
\end{abstract}

Palabras clave: Streptococcus pneumoniae, plantas medicinales, infecciones neomococicas.

\section{Abstract}

Introduction: The healthy carrier is the main disseminator of pneumococcal infections through nasopharynx; an alternative for treatment is a medicinal plant. Objectives: To determine the effectivity of ten medicinal plants against Streptococcus pneumoniae. Design: In vitro experimental study. Biologic materials: Medicinal plant extracts and Streptococcus pneumoniae strains. Interventions: Ten medicinal plant extracts were put in contact in vitro with 10 different Streptococcus pneumonia strains. Main outcome measures: Medicinal plant extracts activity against Streptococcus pneumoniae strains. Results: Only almendro y Bellaco caspi showed small inhibition halos with some strains; with almendro two of 10 Pneumococcus strains were inhibited and with Bellaco caspi seven strains were inhibited and three were resistant. These results had no relation either with Pneumococcus serotype or with antibiotics used according to antibiograms. Conclusions: Bellaco caspi could be an alternative for treating nasopharynx Pneumococcus, but due to resistance to three Streptococcus pneumonia strains there is no total guarantee and an antibiogram for pneumococci against medicinal plants is needed.

Key words: Streptococcus pneumoniae, plants/medicinal, pneumococcal infections.

An Fac med. 2012;73(4):281-3

\section{INTRODUCCIÓN}

El Streptococcus pneumoniae es una bacteria de alta virulencia para el ser humano; es causante de enfermedades que tienen elevada morbilidad y mortalidad en el mundo, que afectan principalmente a niños menores de cinco años y adultos mayores. Ingresa por la nasofaringe y puede causar neumonía, meningitis, bacteriemia, sinusitis, bronquitis, otitis y otros procesos infecciosos.
El portador sano es el principal diseminador de las infecciones neumocócicas por la nasofaringe. En un estudio previo realizado en una guardería infantil se encontró $86 \%$ de niños menores de siete años portadores de neumo$\operatorname{coco}^{(1)}$.

La preocupación principal de los científicos es combatir al Streptococcus pneumoniae en la nasofaringe, ya que las vacunas existentes no cubren la totalidad de los serotipos. Existen varios antibióticos que son usados contra este germen, pero tienen el inconveniente de no ser accesibles a toda la población, el costo alto y el desarrollo de resistencia. Por esta razón, se ha propuesto el uso de plantas medicinales, considerando el conocimiento tradicional que la población peruana tiene al respecto y la gran variedad de plantas medicinales existentes en el territorio nacional.

El presente estudio tuvo como objetivo aplicar in vitro el extracto de ciertas plantas medicinales del Alto Amazonas, para poder probar su efectividad frente a Streptococcus pneumoniae. 


\section{MÉTODOS}

Se realizó un estudio experimental in vitro, para probar la acción antibacteriana de diez plantas medicinales de la región Loreto. El experimento consistió en poner en contacto in vitro cada una de las 10 plantas seleccionadas frente a 10 cepas de Streptococcus pneumoniae criopreservadas.

Las plantas fueron recolectadas en las comunidades nativas de la Provincia de Alto Amazonas, región Loreto, las mismas que fueron identificadas mediante una encuesta a los curanderos tradicionales y a los pobladores de la zona. Las plantas recolectadas fueron:

1. Capirona decorticans (capirona)

2. Coussapoa grandiceps (renaco)

3. Dipteris micranthaharms (Shihual huaco)

4. Duroia hirsuta (Supay chacra)

5. Himatanthus sucuumba (Bellaco caspi)

6. Maranta arundinacea (Shimi pampana)

7. Ocinum micranthum (albahaca)

8. Prunus amygdalus (almendra)

9. Psidium guayaba (guayabo)

10. Solanum kioniotrichum (Suica huito)

La obtención de los extractos se realizó aplicando el siguiente método: las hojas seleccionadas desecadas fueron molidas. Se pesó el molido y se colocó en frascos de color oscuro, con cantidad suficiente de alcohol etílico para cubrirlo totalmente. Se dejó en maceración entre seis y siete días en lugar fresco. Luego, se procedió a filtrar por sistema de vacío. El líquido filtrado fue concentrado en un evaporador rotatorio hasta obtener un residuo semisólido, el cual fue pesado y guardado en recipientes de vidrio color oscuro de boca ancha, etiquetados y cerrados herméticamente.

La prueba in vitro se hizo de la siguiente manera: antes de la experimentación, los extractos de las 10 plantas fueron disueltos individualmente en dimetilsulfóxido (DMSO). Después de obtenida la suspensión, se pasó cada una de ellas por filtro Acrodisc Syringe $25 \mathrm{~mm} \times 0,2$ um de diámetro de los orificios, para decontaminarlos. Luego, se procedió a impregnar discos blancos estériles, sin contenido, con 50 uL de extracto filtrado, por aplicación sucesiva de 20, 15 y $15 \mathrm{uL}$, dejando secar después de cada aplicación por 30 minutos. Luego fueron puestos en contacto, por el método de disco-difusión, con cada una de las 10 cepas de Streptococcus pneumoniae seleccionadas, las cuales se encontraban criopreservadas y fueron reactivadas para el estudio. Las cepas procedieron de las siguientes fuentes: tres aisladas de infecciones localizadas, cuyos serotipos fueron $6 \mathrm{~B}, 19 \mathrm{~F}$ y $23 \mathrm{~F}$; tres cepas multirresistentes, dos con serotipo $19 \mathrm{~F}$ y una $23 \mathrm{~F}$; dos cepas de portadores, con serotipos 14 y $19 \mathrm{~F}$; y dos invasivas, con serotipos 8 y $6 \mathrm{~B}$.

Finalmente, las placas de agar Mueller Hinton, con 5\% de sangre de carnero, con la siembra de Streptococcus pneumoniae y con los discos impregnados con los extractos de las plantas, fueron incubadas a $37^{\circ} \mathrm{C}$ por 48 horas en aerobiosis. La lectura de los resultados consistió en medir el diámetro de los halos de inhibición.

\section{RESULTADOS}

Los extractos de albahaca, guayabo, capirona, supay chacra, shihualhuaco, renaco, suica huito y shimipampana no produjeron halo de inhibición contra las 10 cepas de Streptococcus pneumoniae, como puede apreciarse en la tabla 1.

Almendro presentó pequeños halos de inhibición con dos cepas de Streptococcus pneumoniae, la P-10 (7 mm de diámetro) y la I-6 (9 mm de diámetro); las otras ocho cepas no dieron halo de inhibición (tabla 1).

La inhibición con Bellaco caspi se presentó en siete cepas de Streptococcus pneumoniae. Los halos de inhibición fueron de $10 \mathrm{~mm}$ (MR-4), $9 \mathrm{~mm}$ (IL-7), $8 \mathrm{~mm}$ (IL-1, P-10 e I-6) y $7 \mathrm{~mm}$ (IL12 y P-4). Tres de las cepas no dieron halos de inhibición: MR-2, MR-3 e I-5 $(0 \mathrm{~mm})$ (tabla 1).

Se repitió la prueba con los extractos de almendro y Bellaco caspi contra las 10 cepas y los resultados fueron los mismos.

Las diez cepas de Streptococcus pneumoniae resultaron sensibles a vancomicina, fármaco que se usó como control.

No hubo relación entre los resultados de sensibilidad y resistencia de los 10 extractos con los antibióticos que fueron empleados en los antibiogramas (rifampicina, cefotaxima, ceftriaxona, cefuroxima, lincomicina, ciprofloxacino, eritromicina, cloranfenicol, tetraciclina, penicilina y cotrimoxazol) de Streptococcus pneumoniae realizados en un trabajo anterior ${ }^{(2)}$. Tampoco hubo relación entre los resultados y los serotipos de Streptococcus pneumoniae.

\section{DISCUSIÓN}

Tradicionalmente, los estudios de acción antibacteriana de plantas medicinales se hacen contra una bacteria representante de cada género y especie que se quiere estudiar ${ }^{(3-7)}$. En el presente trabajo, se estudió solo una bacteria, Streptococcus pneumoniae, pero con 10 cepas diferentes que procedían de casos clínicos (infecciones localizadas, sistémicas) y portadores, presentando un perfil de sensibilidad y resistencia diferentes.

Sabiendo que las bacterias mutan frecuentemente para hacerse resistentes a los antibióticos, no podemos afirmar que si una especie bacteriana es sensible a una planta todas sus iguales también lo serán.

Observamos que solo dos plantas presentaron halos de inhibición pequeños, que hace suponer que a mayor concentración del extracto los halos podrían haber sido mayores. Lo sorprendente de los resultados es que hubo cepas de Streptococcus pneumoniae que fueron resistentes tanto al extracto de almendro como al de Bellaco caspi, entendiendo que Streptococcus pneumoniae manifies- 
Tabla 1. Diámetro de los halos de inhibición $(\mathrm{mm})$ de extractos de plantas medicinales con Streptococcus pneumoniae.

\begin{tabular}{|c|c|c|c|c|c|c|c|c|c|c|c|}
\hline $\begin{array}{c}\text { Streptococcus } \\
\text { pneumoniae/Serotipo }\end{array}$ & Albahaca & Almendro & $\begin{array}{l}\text { Bellaco } \\
\text { caspi }\end{array}$ & Guayabo & Capirona & $\begin{array}{l}\text { Supay } \\
\text { chacra }\end{array}$ & Huaca & Renaco & $\begin{array}{l}\text { Suica } \\
\text { huito }\end{array}$ & $\begin{array}{l}\text { Shimi } \\
\text { pampana }\end{array}$ & $\begin{array}{l}\text { Vancomi } \\
\text { cina }\end{array}$ \\
\hline IL-1/6B & 0 & 0 & 8 & 0 & 0 & 0 & 0 & 0 & 0 & 0 & S \\
\hline IL-7/19F & 0 & 0 & 9 & 0 & 0 & 0 & 0 & 0 & 0 & 0 & $S$ \\
\hline IL-12/23F & 0 & 0 & 7 & 0 & 0 & 0 & 0 & 0 & 0 & 0 & $S$ \\
\hline MR-2/19F & 0 & 0 & 0 & 0 & 0 & 0 & 0 & 0 & 0 & 0 & S \\
\hline MR-3/19F & 0 & 0 & 0 & 0 & 0 & 0 & 0 & 0 & 0 & 0 & S \\
\hline MR-4/23F & 0 & 0 & 10 & 0 & 0 & 0 & 0 & 0 & 0 & 0 & $S$ \\
\hline P-4/14 & 0 & 0 & 7 & 0 & 0 & 0 & 0 & 0 & 0 & 0 & $S$ \\
\hline P-10/19F & 0 & 7 & 8 & 0 & 0 & 0 & 0 & 0 & 0 & 0 & $S$ \\
\hline $\mid-6 / 8$ & 0 & 9 & 8 & 0 & 0 & 0 & 0 & 0 & 0 & 0 & $S$ \\
\hline I-15/6B & 0 & 0 & 0 & 0 & 0 & 0 & 0 & 0 & 0 & 0 & $S$ \\
\hline
\end{tabular}

IL: Streptococcus pneumoniae aislado de infección localizada. MR: Streptococcus pneumoniae multirresistentes. P: Streptococcus pneumoniae aislado de portadores sanos. I: Streptococcus pneumoniae aislado de infecciones invasivas. S: Sensible.

ta resistencia al principio activo de estas plantas, aún desconocido, que tiene actividad antineumocócica.

Bellaco caspi es un árbol que tiene flores, frutos y semilla, descrito como un antimicrobiano de amplio espectro ${ }^{(8)}$, por su acción demostrada in vitro contra diferentes bacterias, entre las que no se ha estudiado a Streptococcus pneumoniae. Su mayor uso en la población es la aplicación en heridas infectadas. Sobre el almendro, se menciona que tiene acción antimicrobiana, pero sin especificar los nombres de los microorganismos.

La sensibilidad o resistencia de las cepas de Streptococcus pneumoniae no está vinculada al serotipo. Así mismo, ninguno de los antimicrobianos utilizados en los antibiogramas se comportó in vitro como lo hicieron los dos extractos que tuvieron sensibilidad o resistencia, lo cual nos estaría indicando que los componentes de ambas plantas no tienen relación con los antibióticos conocidos.

En conclusión, Bellaco caspi podría ser una alternativa terapeútica antimicrobiana en la nasofaringe. Pero, por haber presentado resistencia tres cepas de Streptococcus pneumoniae, no se puede garantizar que su efecto sea bueno, por lo que sería conveniente desarrollar un antibiograma de los neumococos contra las plantas medicinales.

\section{AGRADECIMIENTOS}

A la Lic. Carmen Say, quien muy gentilmente realizó la traducción al inglés del resumen.

A la Facultad de Medicina por la subvención económica en el VI Concurso de proyectos de investigación 2009.

Al Dr. Jorge Alarcón Villaverde por la revisión y sugerencias del trabajo.

\section{REFERENCIAS BIBLIOGRÁFICAS}

1. Ponce D, Guevara JM, Valencia E. Prevalencia de colonización de Streptococcus pneumoniae en niños asintomáticos menores de 7 años de un jardin-cuna en Chorrillos, Lima, Perú. IX Congreso Peruano de Enfermedades Infecciosas y Tropicales, Lima-Perú 15-18 de setiembre 2005.

2. Guevara JM, Fenoll A, Valencia E, Zerpa R, Guevara JMM. Streptococcus pneumoniae aislados durante 2002-2006: serotipos y resistencia antibiótica. Correlación con las vacunas existentes. An Fac med. 2008;69(1):29-32
3. Martinez MJ, Molina N, Boucourt E. Evaluación de la actividad antimicrobiana del Psidium guayava (Guayaba). Rev Cubana Plant Med. 1997;2(1):12-4.

4. Cáceres A, Alvarez A, Ovando A, Samayoa B. Plants used in Guatemala for treatment of respiratory diseases. 1. Screening of 68 plants against gram positive bacteria. J Ethnopharmacol. 1991;31(3):193-208.

5. Cruz Mimier C, Espinal G, Castillo D. Sensibilidad y resistencia del Staphylococcus aureus, Haemophilus influenzae, Streptococcus pyogenes frente a cuatro plantas utilizadas en atención primaria de salud por los pobladores del Batey Palavé. Ciencia y Soc. 2008;33(2):153-65.

6. Rérez J, Isaza G, Acosta S. Actividad antibacteriana de extractos de Phenax rugosus y Tabebuia chrysantha. Biosalud Colombia. 2007;6:59-68

7. Dikasso D, Lemma H, Urga K, Debella A, Addis G, Tadele A, Yirsaw K. Investigation on the antibacterial properties of garlic (Allium sativum) on pneumonia causing bacteria. Ethiop Med J. 2002;40(3):241-9.

8. Moreira D, et al. Actividades antimicrobiologicas dos stratos e fracoes obtido atraves de solventes orgânicos da casca da Himathantus sucuuba do vale do Acre. Anais do XV Seminário de iniciação cientifica PIBI Q-CNPQ, 2006: Universidade Federal Do Acre, Rio Branco - Acre. Brazil.

Artículo recibido el 16 de mayo de 2012 y aceptado para publicación el 10 de junio de 2012.

\section{Correspondencia:}

Dr. José Maria Guevara Duncan

Correo electrónico: jomaguedu34@hotmail.com 